\title{
A Novel Application of Multiscale Entropy in Electroencephalography to Predict the Efficacy of Acetylcholinesterase Inhibitor in Alzheimer's Disease
}

\author{
Ping-Huang Tsai, ${ }^{1,2}$ Shih-Chieh Chang,, ${ }^{3,4}$ Fang-Chun Liu, ${ }^{1,2}$ \\ Jenho Tsao, ${ }^{5}$ Yung-Hung Wang, ${ }^{6,7}$ and Men-Tzung Lo ${ }^{6,7}$ \\ ${ }^{1}$ Neurology Department, National Yang-Ming University Hospital, Yi-Lan, Taiwan \\ ${ }^{2}$ Neurology Department, National Yang-Ming University School of Medicine, Taipei, Taiwan \\ ${ }^{3}$ Division of Pulmonology, Department of Internal Medicine, National Yang-Ming University Hospital, Yi-Lan, Taiwan \\ ${ }^{4}$ Department of Internal Medicine, College of Medicine, National Yang-Ming University School of Medicine, Taipei, Taiwan \\ ${ }^{5}$ Graduate Institute of Biomedical Electronics and Bioinformatics, National Taiwan University, Taipei, Taiwan \\ ${ }^{6}$ Center for Dynamical Biomarkers and Translational Medicine, National Central University, Chungli, Taiwan \\ ${ }^{7}$ Research Center for Adaptive Data Analysis, National Central University, No. 300, Jhongda Road, Taoyuan 32001, Taiwan
}

Correspondence should be addressed to Men-Tzung Lo; mzlo@ncu.edu.tw

Received 1 December 2014; Accepted 2 January 2015

Academic Editor: Kazeem O. Okosun

Copyright (C) 2015 Ping-Huang Tsai et al. This is an open access article distributed under the Creative Commons Attribution License, which permits unrestricted use, distribution, and reproduction in any medium, provided the original work is properly cited.

\begin{abstract}
Alzheimer's disease (AD) is the most common form of dementia. According to one hypothesis, AD is caused by the reduced synthesis of the neurotransmitter acetylcholine. Therefore, acetylcholinesterase (AChE) inhibitors are considered to be an effective therapy. For clinicians, however, AChE inhibitors are not a predictable treatment for individual patients. We aimed to disclose the difference by biosignal processing. In this study, we used multiscale entropy (MSE) analysis, which can disclose the embedded information in different time scales, in electroencephalography (EEG), in an attempt to predict the efficacy of AChE inhibitors. Seventeen newly diagnosed AD patients were enrolled, with an initial minimental state examination (MMSE) score of $18.8 \pm 4.5$. After 12 months of AChE inhibitor therapy, 7 patients were responsive and 10 patients were nonresponsive. The major difference between these two groups is Slope 2 (MSE6 to 20). The area below the receiver operating characteristic (ROC) curve of Slope 2 is $0.871(95 \% \mathrm{CI}=0.69-1)$. The sensitivity is $85.7 \%$ and the specificity is $60 \%$, whereas the cut-off value of Slope 2 is -0.024 . Therefore, MSE analysis of EEG signals, especially Slope 2, provides a potential tool for predicting the efficacy of AChE inhibitors prior to therapy.
\end{abstract}

\section{Introduction}

Alzheimer's disease (AD) is the most common form of dementia $[1,2]$, with the dominant presentation of a progressive decline in cognitive functioning beyond what is expected from normal aging. The neurodegeneration in $\mathrm{AD}$ may be caused by deposition of amyloid beta-peptide in plaques or formation of neurofibrillary tangle in brain tissue $[1,2]$. Although little is known of the actual cause of $\mathrm{AD}$, many of its symptoms are generally accepted to be related to a cholinergic deficit in the cerebral cortex and other areas of the brain [3-5]. Acetylcholinesterase (AChE) inhibitors, which inhibit the acetylcholinesterase enzyme from breaking down acetylcholine and thereby increasing the level and duration of the neurotransmitter acetylcholine activity, have been proven to be an effective therapy for $\mathrm{AD}$ [6-11]. Pharmacoeconomic studies have demonstrated that therapies can postpone dementia from progressing to more severe stages and may also result in economic benefits for patients' families and caregivers, as well as for society [12-16]. However, clinicians have argued that AChE inhibitors have an effect on a subgroup of only $25-50 \%$ of AD patients [1719], which cannot be identified objectively prior to therapy. Furthermore, the time scale for measuring the effect of AChE 
inhibitors can last anywhere between several months to several years.

Recently, numerous studies have attempted to identify a prognostic predictor of $\mathrm{AD}$ by using artificial neural networks [20], brain magnetic resonance imaging (MRI) [18, 21], single-photon emission computed tomography (SPECT) [22], and cognitive function tests [23]. However, technical dependence, high costs, contrast-agent related allergies, and potential exposure to radionuclide irradiation have limited their clinical application. On the other hand, numerous forms of quantitative electroencephalographic (EEG) analyses have been used to elucidate the characteristics of EEGs to enhance diagnostic power with the assistance of signal processing, suggesting a potential objective tool in the evaluation of AD [24-36]. The surface EEG represents the electrical activity of innumerable cortical neurons, which determine the fundamental patterns that indicate the interaction between various mechanisms with multiple and spatial scales. As it is quite difficult to explain the underlying neurophysiology mechanism, nonlinear based methods have recently been used more frequently to explore the EEG activity [37, 38]. The majority of the studies that have used nonlinear methods showed the loss of the complexity of EEG signals to be correlated with the severity of the dementia [31, 32, 34, 35]. However, it is difficult to determine which specific physiological mechanism degrades the complexity of the EEG, and, as a result, clinicians lack information regarding the effective responder to $\mathrm{AChE}$ inhibitors in $\mathrm{AD}$ patients.

In light of fundamental nonlineal theory, biological signals represent the outcome of the nonlinear interactions between different processes at multiple temporal and spatial scales, including EEGs and electrocardiograms (ECG). With this understanding, some studies proposed a careful examination of changes in nonlinear indices with scales. The most well-known of these changes is the crossover phenomenon of the fractal correlation exponents between short and long time scales in the detrended fluctuation analysis (DFA) [39] of heart-rate dynamics. The short-term exponent is understood to be determined mostly by cardiorespiratory interaction [39, 40]. Recently, the studies of activity fluctuation with aging and in $\mathrm{AD}[41,42]$ determined that fractal correlations at certain scales (i.e., 1.5-8.0 hours) declined with age. These studies also determined that an age-independent AD effect further reduced the correlations at these scales, leading to the greatest reduction of the correlations in very old people with late-stage $\mathrm{AD}$. This result closely resembles the loss of correlations at long time scales in suprachiasmatic nucleus- (SCN-) lesioned animals [43]. In addition to DFA, multiscale entropy (MSE) analysis, proposed by Costa et al. $[44,45]$, is a possible method for measuring the complexity of nonlineal signals at different temporal scales by means of an entropy-based algorithm. In our previous study, we proposed a detrending process $[31,46,47]$ to attenuate the spurious influence caused by nonstationarity in the real world. This study also demonstrated many features of the scale function of entropy, including the slope of the scale function of entropy used to check the existence of fractal correlation (a negative slope indicates a greater number of random fluctuations corresponding to loss of interaction), which can assist with clinical classification [47]. For example, sympathetic and parasympathetic activities are correlated with MSE on different scales of the heartbeat sequence (scales 3-5 and 1-4 for sympathetic activity and parasympathetic activity, resp.) [46]. Furthermore, congestive heart failure patients without $\beta$-blocker therapy exhibit a very negative slope for MSE1-5, indicating the lack of interaction to be a result of respiratory regulation [40].

Tracing the activation of the neurotransmitter ACh, which is released from the presynaptic vesicles that are triggered by action potential (electrical impulse), diffused in the synapse, and then finally conjugated to the postsynaptic receptor (5-50 msec) [48-50], these activations are observed at different time scales, each of which may be studied using MSE analysis.

In this study, we hypothesize that some features, such as slope, of MSE analysis of EEG data can be associated with the therapy efficacy of AChE inhibitors in AD patients, relying on the ability of MSE to demonstrate different mechanisms with multiple temporal or spatial scales.

\section{Materials and Methods}

2.1. Participants. All participants in this study were diagnosed with dementia and enrolled from the neurological clinic at the National Yang Ming University Hospital. After screening for dementia, all participants received further medical, neurological, neuropsychological, and psychiatric assessments, as well as blood examinations. Neurological assessments for all participants included cerebral computed tomography (CT) scans to rule out intracranial pathology (i.e., brain tumors or stroke) that may have contributed to cognitive decline. Trained research assistants administered the Chinese version of the minimental state examination (MMSE) [51], which features a total score of 30. The clinical dementia rating (CDR) was also used to determine the severity of dementia after a neurologist conducted separate semistructured interviews with the patient and a knowledgeable informant. The scores are as follows: 0 for normal, 0.5 for questionable, 1 for mild, 2 for moderate, and 3 for severe [52]. $\mathrm{AD}$ was diagnosed according to the diagnostic criteria of the Diagnostic and Statistical Manual of Psychiatric Disorders, 4 th revised [53]. EEG was not included as part of the routine workup.

The inclusion criteria for participation in the EEG study included (1) a diagnosis of probable $\mathrm{AD}$ based on the Diagnostic and Statistical Manual of Psychiatric Disorders, 4 th revised (DSM-IV) criteria [53] and (2) a rating of either 1 or 2 on the CDR scale [52]. Patients with dementia that may have been caused by reasons other than $\mathrm{AD}$, as determined by the patient's history, neurological examinations, imaging, and blood studies, were excluded from this study so as to ensure that alterations in EEGs were caused exclusively by $\mathrm{AD}$. The research was approved by the institutional review board of National Yang-Ming University Hospital, YiLan, Taiwan, a local community teaching hospital. Written informed consent was obtained from each participant prior to the study. 
Seventeen newly diagnosed AD patients ( 9 men and 8 women, mean age $74.6 \pm 7.4$ ) were enrolled in this study, with an initial MMSE score of $18.8 \pm 4.5$. Two of these patients were moderately demented $(\mathrm{CDR}=2)$, whereas the remaining 15 were mildly demented $(C D R=1)$. None of the patients received any antipsychotics prior to the first EEG. Each participant received the AChE inhibitor donepezil hydrochloride (Aricept) $5 \mathrm{mg} / \mathrm{d}$ for 12 months and follow-up MMSE 12 months after therapy.

2.2. EEG Recordings. EEGs were recorded for all subjects. In accordance with International Federation of Clinical Neurophysiology (IFCN) standards for the digital recording of clinical EEG [54], the surface EEG signals were recorded using a digital EEG recorder (NicoletOne) from the 19 scalp loci of the international 10-20 system (channels Fp1, Fp2, F3, F4, C3, C4, P3, P4, O1, O2, F7, F8, T3, T4, T5, T6, Fz, Cz, and $\mathrm{Pz}$ ), with all electrodes referenced to the bilateral ear (A1, A2) for $30 \mathrm{~min}$. ECGs were recorded on a separate channel as a part of EEG recordings. The sample frequency was $256 \mathrm{~Hz}$ and the analog-to-digital converter digitalized resolution was 12 bits. All the patients remained awake in a relaxed state in order to undergo a 30 min eyes-closed EEG.

\subsection{Signal Processing and Analysis: Multiscale Entropy Anal-} ysis. We adopted MSE as a complexity measure to feature digitalized EEG signals. Before conducting the analysis, one $30 \mathrm{sec}$ artifact-free epoch EEG signal was selected by an experienced neurologist. Furthermore, empirical mode decomposition [55], which is considered superior to the traditional linear quantitative method to ascertain the intrinsic complexity, was utilized to remove the trend $(<1 \mathrm{~Hz})$ from the signals as was also the case in our previous study [31]. The MSE method is comprised of the following two steps: (1) coarse-graining the signals into different time scales and (2) quantifying the degree of irregularity in each coarse-grained time series using sample entropy (SampEn) [56, 57].

2.3.1. Sampling Entropy (SampEn). $\left\{x_{i}\right\}=\left\{x_{1}, x_{2}, \ldots, x_{i}, \ldots\right.$, $\left.x_{N}\right\}$ represents a time series of data length $N$. The $m$-length vector extracted from time series $\left\{x_{i}\right\}$ was defined by $u_{m}(i)=$ $\left\{x_{i}, x_{i+1}, \ldots, x_{i+m-1}\right\}$ where $n_{i}^{m}(r)$ represents the number of vectors, $u_{m}(j)$ that are close to the given vector, $u_{m}(i)$. To obtain $n_{i}^{m}(r)$, we first defined the distance, $d\left[u_{m}(i), u_{m}(j)\right]$ between the given vector $u_{m}(i)$ and $u_{m}(j)$ as the maximum absolute difference between their corresponding scalar elements, that is, $d\left[u_{m}(i), u_{m}(j)\right]=\max _{k=0, m-1}\left[\left|x_{i+k}-x_{j+k}\right|\right]$. Then, $n_{i}^{m}(r)$ was defined by counting the number of $u_{m}(j)$ with $\left.d\left[u_{m}(i), u_{m}(j)\right]\right|_{j=1, n-m+1}$ smaller than the threshold, $r$, a given prior. Note that here $j \neq i$; that is, self-matches were not included to avoid the bias of ApEn [58]. The ratio of $n_{i}^{m}(r)$ to total number of $m$-vectors extracted from the time series was evaluated, and the result was denoted as $C_{r}^{m}(i)$. The above steps were repeated to find $C_{r}^{m}(i)$ from $i=1$ to $i=N-m+1$; then the natural logarithm of each $C_{r}^{m}(i)$ was calculated and then averaged over $i$ to obtain $\phi^{m}(r)$. Theoretically, sample entropy [56, 57] is defined as $\operatorname{SampEn}(m, r)=\phi^{m}(r)-$ $\phi^{m+1}(r)$. Note that the average of $C_{r}^{m}(i)$ can be regarded as the probability that any two vectors extracted from time series $\left\{x_{i}\right\}$ are similar in some sense; therefore, $\phi^{m}(r)-\phi^{m+1}(r)$ and conceptually represents the average of the natural logarithm of the conditional probability that the sequences that are close to each other for $m$ consecutive data points will still be close to each other when one more point is given.

2.3.2. Multiscale Entropy (MSE). SampEn is a statistical measure based on information theory used to quantify the irregularity of a sequence of data [56-58]. It examines a time series for occurrences of similar epochs of a preassigned length; similarity is determined with respect to whether the distance between epochs is under a tolerance $r$ or not. More specifically, it calculates the negative natural logarithm of the conditional probability (i.e., those epochs) similar for $m$ points that will remain so when one additional point is added to the subseries [56-58]. The higher the SampEn is, the more unpredictable the data sequence is. However, complicated systems typically generate highly irregular output [37] and also exhibit dynamically diverse tendencies on various time scales, including, for example, the coexistence of slow and fast phenomena. Although SampEn provides an adequate way of quantifying irregularities for physiological output, it is not sufficient alone to cope with the complex content for the purpose of physiological research. In contrast, MSE analysis, based on evaluating the sample entropy at multiple time scales, has proven useful in this regard. To recast a signal in another scale, the original series were segregated into blocks, where each block contained $n$ data points. The mean value over each block then formed the coarse-grained time series at scale $n$. It is clear that the time series at coarse-grained scale 1 is identical to the original signal. Equipped with multiple scales, the MSE method can disclose the temporal structures as well as scale-dependent characteristics of signals.

2.3.3. Slope of MSE. In this study, the MSE value of each lead was calculated individually, which amounts to 380 values for each patient. Slopes of the mean value of MSE, averaged out of all leads, over scales 1-5 (Slope 1) and 6-20 (Slope 2) were estimated using the least-squares method (i.e., one Slope 1 and one Slope 2 for each patient). Throughout the analysis, we set $m=2$ and $r=0.15$ times the standard deviation of the processed signal, as was the case in our previous study [31].

2.4. Statistical Analyses. Based on the differences between the follow-up and the initial MMSE scores, the patients were divided into two groups: a responder group and a nonresponder group. A responder was identified as a patient whose follow-up MMSE score was greater than or equal to the initial score; patients whose follow-up MMSE scores were less than the initial score were classified as nonresponders.

Descriptive statistics were presented as means \pm standard deviation (SD). SPSS (ver16.0) for Windows (SPSS Inc., Chicago, IL, USA) was used for analysis. Baseline demographic characteristics, including age, MMSE, CDR, and MSE of each lead, and Slope 1 and Slope 2 were coded as continuous variables. Other demographic characteristics, such as sex, were coded as dichotomous variables. All of 
these characteristics were treated as predicable variables for treatment response. Because of the small sample size in this study, a Mann-Whitney $U$ test (instead of Student's $t$-test) was used to determine which factors were significant. A forward logistic regression, being a regression model in which the choice of predictive variables is carried out by an automatic procedure, which involves starting with no variables in the model, testing the addition of each variable using a chosen model comparison criterion, adding the variable (if any) that improves the model the most, and repeating this process until none improves the model was then used to calculate the odds ratio in the variants. All of the statistical tests were two-tailed and significance levels were set at a $P$ value of less than 0.05 .

By conducting the receiver operating characteristic (ROC) analysis and considering the optimal combination of sensitivity and specificity, we determined the best cut-off points. Likelihood ratios and positive and negative predictive values with 95\% confidence intervals (CI) were assessed at each of the cut-off point levels.

\section{Results}

Seven patients $(3 \mathrm{M} / 4 \mathrm{~F}$, mean age $76.1 \pm 7.9)$ were categorized as responders and ten patients $(6 \mathrm{M} / 4 \mathrm{~F}$, mean age $73.5 \pm 7.3)$ were categorized as nonresponders. There were no significant differences in age and gender between the patients in these two groups. The initial MMSE score in the responder group was 15.6 \pm 5.1 , and in the nonresponder group it was $20.9 \pm 2.8$ $(P=0.070)$. The MSE values in each lead were significantly higher in F7 MSE7, Fz MSE6, Fz MSE7, Fz MSE8, C4 MSE5, T4 MSE6, T4 MSE7, T4 MSE9, Pz MSE7, Pz MSE8, and O1 MSE7 (Table 1). Slope 1 was $0.063 \pm 0.038$ for the responder group and $0.092 \pm 0.071$ for the nonresponder group $(P=$ 0.333 ) (Table 1 and Figure 1). Slope 2 was $-0.008 \pm 0.019$ for the responder group and $-0.03 \pm 0.009$ for the nonresponder group $(P=0.021)$ (Table 1 and Figure 1$)$. After applying the forward logistic regression, only Slope 2 was preserved. The other factors, including the initial MMSE, were excluded.

The area under the ROC curve of Slope 2 (Figure 2) was $0.871(95 \% \mathrm{CI}=0.69-1)$. The sensitivity was $85.7 \%$ and the specificity was $60 \%$, while the cut-off value of Slope 2 was -0.024 (Figure 3).

\section{Discussion}

This pilot study demonstrated the potential of quantitative EEG, the slope of MSE, to predict the efficacy of AChE inhibitors in treating AD. When Slope 2 (MSE 6-20) is less than -0.024 , therapy efficacy is relatively poor, with a sensitivity of $85.7 \%$ and specificity of $60 \%$. However, the efficacy of AChE inhibitors in $\mathrm{AD}$ was affected by various factors, including antipsychiatric agents for behavior and psychiatric syndromes, other systemic disorders, hypertension, diabetes, and so on. Therefore, it is difficult to predict the efficacy of AChE inhibitors using a single biomarker, such as MSE in this study. However, this study can produce other findings, including whether or not the dosage of the AChE inhibitor is sufficient and whether we should try a form of
TABLE 1: The demographic characteristics of two groups.

\begin{tabular}{lccc}
\hline & Responder & Nonresponder & $P$ value \\
\hline Age & $76.1 \pm 7.9$ & $73.5 \pm 7.3$ & 0.669 \\
Sex & $3 \mathrm{M} / 4 \mathrm{~F}$ & $6 \mathrm{M} / 4 \mathrm{~F}$ & 0.601 \\
MMSE & $15.9 \pm 5.1$ & $20.9 \pm 2.8$ & 0.070 \\
CDR & $0.8 \pm 0.6$ & $1.2 \pm 0.6$ & 0.230 \\
Slope 1 & $0.063 \pm 0.038$ & $0.092 \pm 0.071$ & 0.475 \\
Slope 2 & $-0.008 \pm 0.019$ & $-0.03 \pm 0.009$ & $0.010^{*}$ \\
F7 MSE7 & $1.85 \pm 0.15$ & $1.98 \pm 0.10$ & $0.043^{*}$ \\
Fz MSE6 & $1.84 \pm 0.26$ & $2.04 \pm 0.06$ & $0.025^{*}$ \\
Fz MSE7 & $1.87 \pm 0.23$ & $2.04 \pm 0.08$ & $0.043^{*}$ \\
Fz MSE8 & $1.84 \pm 0.21$ & $2.01 \pm 0.09$ & $0.033^{*}$ \\
C4 MSE5 & $1.84 \pm 0.25$ & $2.05 \pm 0.10$ & $0.043^{*}$ \\
T4 MSE6 & $1.77 \pm 0.21$ & $1.99 \pm 0.10$ & $0.019^{*}$ \\
T4 MSE7 & $1.79 \pm 0.20$ & $1.99 \pm 0.12$ & $0.033^{*}$ \\
T4 MSE9 & $1.79 \pm 0.22$ & $2.00 \pm 0.16$ & $0.019^{*}$ \\
Pz MSE7 & $1.92 \pm 0.20$ & $2.13 \pm 0.10$ & $0.010^{*}$ \\
Pz MSE8 & $1.95 \pm 0.17$ & $2.13 \pm 0.10$ & $0.007^{*}$ \\
O1 MSE7 & $1.89 \pm 0.23$ & $2.08 \pm 0.10$ & $0.033^{*}$ \\
\hline Dem
\end{tabular}

Demographic characteristics of two groups, including age, sex, MMSE, CDR, Slope 1, Slope 2, and MSE values of each lead with significant differences in Mann-Whitney $U$ test. ${ }^{*}$ Indication that the correlation is significant at the 0.05 level (2-tailed).

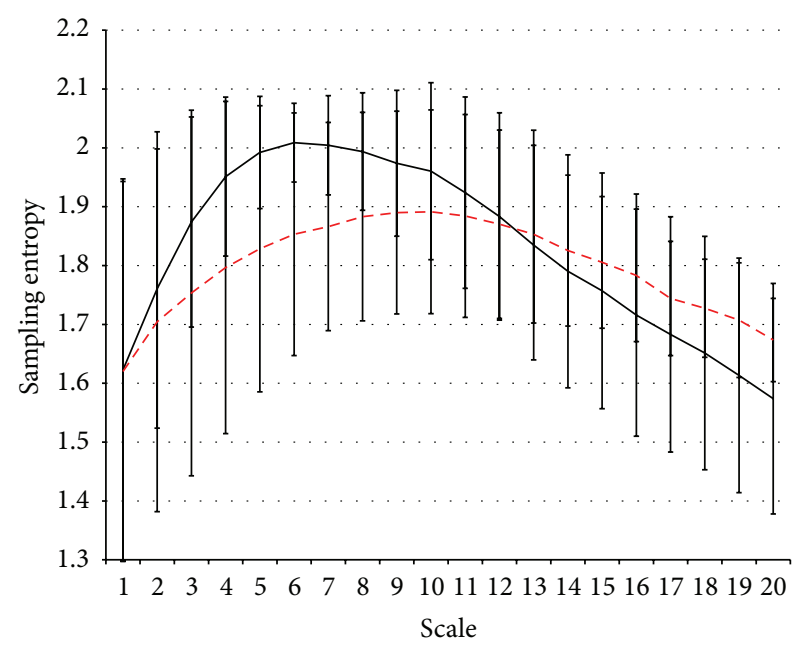

FIGURE 1: The mean value of MSE in the responder and nonresponder. The red dashed line indicates the responder and the black solid line indicates nonresponders.

combination therapy (i.e., AChE inhibitor and N-methyl-daspartate (NMDA) receptor antagonist) for patients with a very negative slope at the region with scales 6-20 (i.e., Slope 2).

Understanding the complexity at certain scales may correspond to the illness of specific physiology processes; furthermore, MSE analysis of EEG signals is a possible way to profile the cholinergic effect in cortex. Figure 1 illustrates the maximum value of MSE which occurred at MSE6 (approximately $20 \mathrm{msec}$ in the time scale) in nonresponders and almost a plateau from MSE5 to MSE10 (approximately 


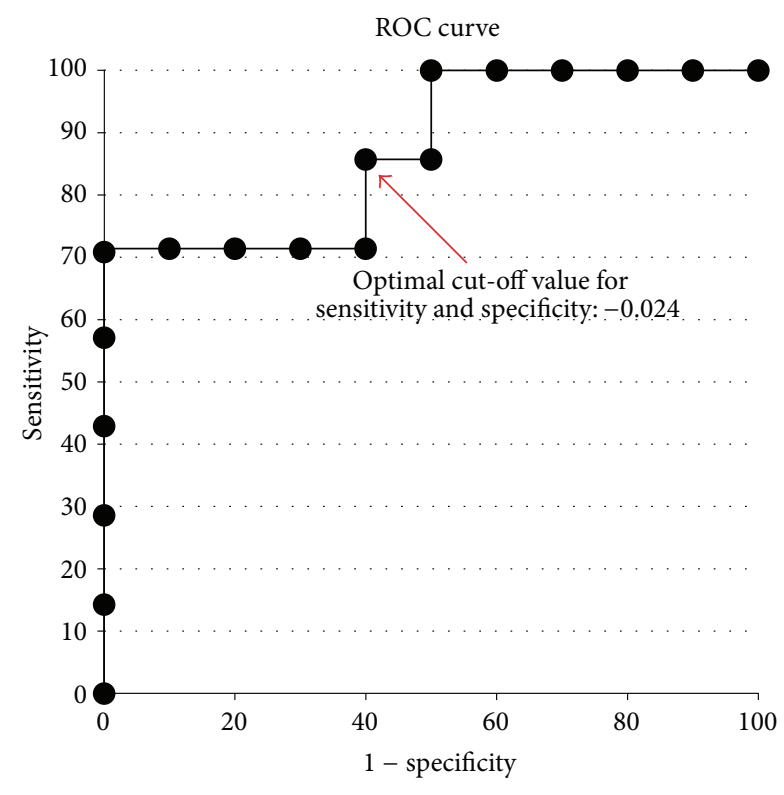

FIGURE 2: The ROC curve of Slope 2.

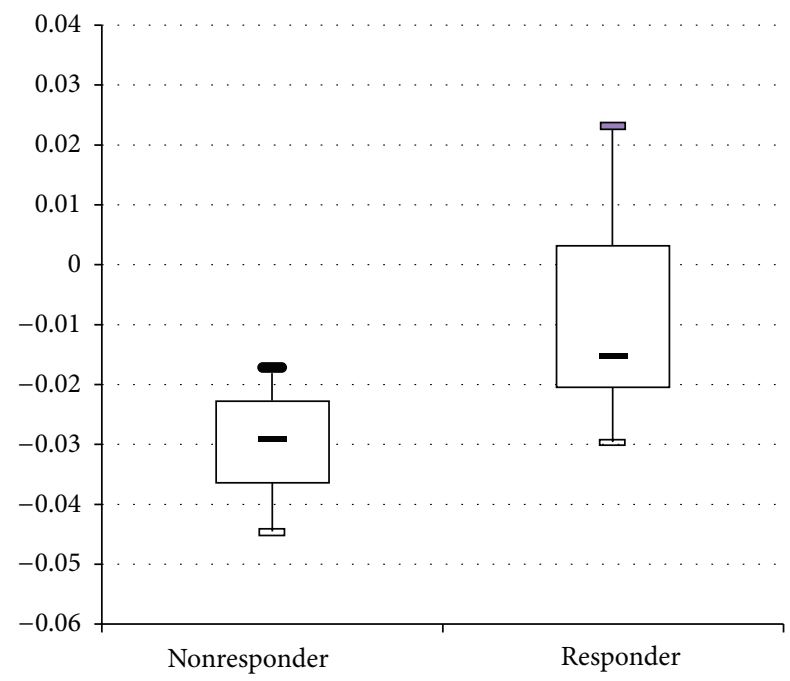

FIGURE 3: The distributions of Slope 2 in responders and nonresponders.

$20 \mathrm{msec}$ to $40 \mathrm{msec}$ in the time scale) in responders. This indicates that time scales are compatible with the transfer time from the presynapse to the postsynapse, where the membranes are separated by a synaptic cleft that averages 20 to $50 \mathrm{~nm}(0.02$ to $0.05 \mu \mathrm{m})$ in width $[50,59]$ and lasts for approximately $15 \mathrm{msec}$ [48-50]. Furthermore, it takes approximately $20 \mathrm{msec}$ to break the ACh molecules from the binding receptor sites [48]. For the sampling rate of $256 \mathrm{~Hz}$, the small (1-5) and large scales (6-20) of MSE could be associated with transfer time from the presynapse to postsynapse and binding time of ACh molecules, respectively. Therefore, the features of MSE1 to MSE5 are responsive to the amount of released ACh or the concentration of ACh in the synapse. In addition, the higher value in F7 MSE7, Fz MSE6,
Fz MSE7, Fz MSE8, C4 MSE5, T4 MSE6, T4 MSE7, T4 MSE9, $\mathrm{Pz}$ MSE7, Pz MSE8, and O1 MSE7 in nonresponders may be attributed to the compensative release of ACh to maintain a similar cognitive activation as the responder. In the larger scales $(>12)$, the nonresponders have smaller values than the responders. However, this difference is not significant statistically (Figure 1), which suggests the poor binding ability of ACh to the receptor. On the other hand, the significantly negative slope (the region with scales over $20 \mathrm{msec}$ ) of the scale function of entropy for the nonresponders indicates the disrupted fractal pattern (i.e., more random fluctuations) and thus suggests the reduction of interaction in the neurological network (working at time scales over $20 \mathrm{msec}$ ). We can hypothesize that the slope of MSE in large time scales $(n \geq$ 6) is responsive to the Ach molecular binding ability or binding count; the nonresponders have lower ACh molecular binding ability or binding count and poor interaction in the neurological network. They therefore may require a higher dose of AChE inhibitors or other mechanism therapy, such as NMDA receptor antagonist, to improve clinical syndromes. A study by Escudero et al. [37] showed the slope of MSE in long time scale $(n \geq 6)$ is larger in normal control, which is compatible with our hypothesis.

Previous studies $[37,38]$ showed a higher complexity in demented patients in long time scales, which challenges the above hypothesis as the long time scales are related to the ACh molecular binding ability or binding counts. However, there have been some significant differences with regards to methods and study design, such as the severity of demented patients in this study and empirical mode decomposition (EMD) as a preprocessing to detrend the signals, which would produce better results in nonstationary physiology signals [31]. Nevertheless, other neurophysiological mechanisms might exist to explain both conditions.

The initial MMSE in the responders showed lower scales than that of the nonresponders (although not significant statistically), and this may be the reason why there is a higher response rate to $\mathrm{AChE}$ inhibitors among the responders. However, the usefulness of MMSE was excluded by following forward logistic regression. A previous study [23] showed no significant difference in pretreatment MMSE scores between responders and nonresponders, which is a result that is similar to the one in our study. In that same study, better visual-spatial motor ability, clock drawing, and tracking test results were also recorded among the responders. Therefore, the lower MMSE score among responders in this study does not explain the difference between responders and nonresponders.

MSE analyses of EEG data in this study disclose the different Slope 2 value in responders and nonresponders for AChE inhibitors, indicating an existing physiologic condition, which confirms our hypothesis regarding the neurotransmitter, Ach, or other nonlinear interactions between different processes at multiple temporal and spatial scales. More rigorous study is required to elucidate the physiological significance of the values and slopes of MSE. Due to a lack of other types of medication in this study, the application of predicting the efficacy with respect to different types of AChE inhibitors or NMDA antagonist is also uncertain. 
TABLE 2: The raw data of two groups; MMSE as minimental status examination; CDR as clinical dementia rating.

\begin{tabular}{lccc}
\hline Group & Age & MMSE & CDR \\
\hline Nonresponder & 65 & 25 & 1 \\
Nonresponder & 80 & 19 & 1 \\
Nonresponder & 81 & 20 & 1 \\
Nonresponder & 61 & 24 & 1 \\
Nonresponder & 80 & 21 & 1 \\
Nonresponder & 73 & 24 & 1 \\
Nonresponder & 71 & 16 & 1 \\
Nonresponder & 67 & 21 & 1 \\
Nonresponder & 81 & 19 & 1 \\
Nonresponder & 76 & 20 & 1 \\
Responder & 69 & 21 & 1 \\
Responder & 67 & 21 & 1 \\
Responder & 88 & 16 & 1 \\
Responder & 71 & 14 & 1 \\
Responder & 84 & 9 & 2 \\
Responder & 79 & 10 & 2 \\
Responder & 75 & 20 & 1 \\
\hline
\end{tabular}

Lastly, although this study was limited by its small sample size, the statistical power was still high enough to support our findings.

\section{Conclusion}

MSE analysis of EEG recordings can show characteristics both at short and at long time scales and provide a potential tool for predicting the efficacy of AChE inhibitors in AD. This nonlineal method improved EMD-based sampling entropy, which was introduced as an optimum method for evaluating embedded information in EEG and as an objective, noninvasive, and cost-effective tool for evaluating and monitoring $\mathrm{AD}$ patients [31], but not for providing enough information about the possible responder to the AChE inhibitor in $\mathrm{AD}$.

\section{Appendix}

See Table 2.

\section{Conflict of Interests}

The authors declare that there is no conflict of interests regarding the publication of this paper.

\section{Acknowledgments}

The research protocol was funded by a grant (RD2009-010) from the National Yang-Ming University Hospital, Yi-Lan, Taiwan. Dr. Men-Tzung Lo was supported by the National Science Council (NSC; Taiwan, ROC), Grant no. 100-2221E-008-008-MY2; a joint foundation of Cathay General Hospital and National Central University, Grant no. CNJRF99CGH-NCU-A3; a joint foundation of Taipei Veterans
General Hospital and National Central University, Grant no. VGHUST100-G1-4-3; and NSC supports from the Center for Dynamical Biomarkers and Translational Medicine, National Central University, Taiwan (NSC 100-2911-I-008-001).

\section{References}

[1] B. J. Sadock, V. A. Sadock, and H. I. Kaplan, Kaplan and Sadock's Comprehensive Textbook of Psychiatry, Lippincott Williams \& Wilkins, 2005.

[2] L. P. Rowland and T. A. Pedley, Merritt's Neurology, Lippincott Williams \& Wilkins, 2009.

[3] P. Davies and A. J. F. Maloney, "Selective loss of central cholinergic neurons in Alzheimer's disease," The Lancet, vol. 2, no. 8000, p. 1403, 1976.

[4] R. E. Davis, P. D. Doyle, R. T. Carroll, M. R. Emmerling, and J. Jaen, "Cholinergic therapies for Alzheimer's disease. Palliative or disease altering?" Arzneimittel-Forschung, vol. 45, no. 3, pp. 425-431, 1995.

[5] E. Giacobini, "Pharmacotherapy of Alzheimer disease: new drugs and novel strategies," Progress in Brain Research, vol. 98, pp. 447-454, 1993.

[6] S. M. Greenberg, M. K. Tennis, L. B. Brown et al., "Donepezil therapy in clinical practice: a randomized crossover study," Archives of Neurology, vol. 57, no. 1, pp. 94-99, 2000.

[7] S. L. Rogers, "Perspectives in the management of Alzheimer's disease: clinical profile of donepezil," Dementia and Geriatric Cognitive Disorders, vol. 9, supplement 3, pp. 29-42, 1998.

[8] S. L. Rogers, R. S. Doody, R. C. Mohs, and L. T. Friedhoff, "Donepezil improves cognition and global function in Alzheimer disease: a 15-week, double-blind, placebo-controlled study," Archives of Internal Medicine, vol. 158, no. 9, pp. 10211031, 1998.

[9] S. L. Rogers, M. R. Farlow, R. S. Doody, R. Mohs, and L. T. Friedhoff, "A 24-week, double-blind, placebo-controlled trial of donepezil in patients with Alzheimer's disease," Neurology, vol. 50, no. 1, pp. 136-145, 1998.

[10] S. L. Rogers and L. T. Friedhoff, "The efficacy and safety of donepezil in patients with Alzheimer's disease: results of a US multicentre, randomized, double-blind, placebo-controlled trial. The Donepezil Study Group," Dementia, vol. 7, no. 6, pp. 293-303, 1996.

[11] B. Winblad, L. Kilander, S. Eriksson et al., "Donepezil in patients with severe Alzheimer's disease: double-blind, parallel-group, placebo-controlled study," The Lancet, vol. 367, no. 9516, pp. 1057-1065, 2006.

[12] H. Fillit and J. Hill, "Economics of dementia and pharmacoeconomics of dementia therapy," The American Journal Geriatric Pharmacotherapy, vol. 3, no. 1, pp. 39-49, 2005.

[13] G. B. Frisoni, "Reimbursement of acetilcholinesterase inhibitors for Alzheimer's disease in Europe," International Journal of Geriatric Psychiatry, vol. 16, no. 2, pp. 233-235, 2001.

[14] D. Getsios, S. Blume, K. J. Ishak, and G. D. H. Maclaine, "Cost effectiveness of donepezil in the treatment of mild to moderate Alzheimer's disease: a UK evaluation using discreteevent simulation," PharmacoEconomics, vol. 28, no. 5, pp. 411427, 2010.

[15] B. R. Williams, A. Nazarians, and M. A. Gill, "A review of rivastigmine: a reversible cholinesterase inhibitor," Clinical Therapeutics, vol. 25, no. 6, pp. 1634-1653, 2003. 
[16] J. L. Fun and S. J. Wang, "Cost-effectiveness analysis of donepezil for mild to moderate Alzheimer's disease in Taiwan," International Journal of Geriatric Psychiatry, vol. 23, no. 1, pp. 73-78, 2008.

[17] E. Giacobini, "Cholinesterase inhibitor therapy stabilizes symptoms of Alzheimer disease," Alzheimer Disease and Associated Disorders, vol. 14, supplement 1, pp. S3-S10, 2000.

[18] H. Hanyu, Y. Tanaka, H. Sakurai, M. Takasaki, and K. Abe, "Atrophy of the substantia innominata on magnetic resonance imaging and response to donepezil treatment in Alzheimer's disease," Neuroscience Letters, vol. 319, no. 1, pp. 33-36, 2002.

[19] H. Kaduszkiewicz, T. Zimmermann, H.-P. Beck-Bornholdt, and H. D. van Bussche, "Cholinesterase inhibitors for patients with Alzheimer's disease: systematic review of randomised clinical trials," British Medical Journal, vol. 331, no. 7512, pp. 321-327, 2005.

[20] P. Mecocci, E. Grossi, M. Buscema et al., "Use of artificial networks in clinical trials: a pilot study to predict responsiveness to donepezil in Alzheimer's disease," Journal of the American Geriatrics Society, vol. 50, no. 11, pp. 1857-1860, 2002.

[21] H. Kanetaka, H. Hanyu, K. Hirao et al., "Prediction of response to donepezil in Alzheimer's disease: combined MRI analysis of the substantia innominata and SPECT measurement of cerebral perfusion," Nuclear Medicine Communications, vol. 29, no. 6, pp. 568-573, 2008.

[22] J. Hongo, S. Nakaaki, Y. Shinagawa et al., "SPECT-identified neuroanatomical predictor of the cognitive effects of donepezil treatment in patients with Alzheimer's disease," Dementia and Geriatric Cognitive Disorders, vol. 26, no. 6, pp. 556-566, 2008.

[23] D. Saumier, S. Murtha, H. Bergman, N. Phillips, V. Whitehead, and H. Chertkow, "Cognitive predictors of donepezil therapy response in alzheimer disease," Dementia and Geriatric Cognitive Disorders, vol. 24, no. 1, pp. 28-35, 2007.

[24] R. Chiaramonti, G. C. Muscas, M. Paganini et al., "Correlations of topographical EEG features with clinical severity in mild and moderate dementia of Alzheimer type," Neuropsychobiology, vol. 36, no. 3, pp. 153-158, 1997.

[25] P. Etévenon, P. Péron-Magnan, B. Gueguen, M. Ghanem, J. Gaches, and P. Deniker, "Value of quantitative EEG and EEG mapping in medicine," Annales de Medecine Interne, vol. 138, no. 1, pp. 13-18, 1987.

[26] V. Knott, A. Labelle, B. Jones, and C. Mahoney, "Quantitative EEG in schizophrenia and in response to acute and chronic clozapine treatment," Schizophrenia Research, vol. 50, no. 1-2, pp. 41-53, 2001.

[27] G. Rodriguez, P. Vitali, M. Canfora et al., "Quantitative EEG and perfusional single photon emission computed tomography correlation during long-term donepezil therapy in Alzheimer's disease," Clinical Neurophysiology, vol. 115, no. 1, pp. 39-49, 2004.

[28] G. G. Yener, A. F. Leuchter, D. Jenden, S. L. Read, J. L. Cummings, and B. L. Miller, "Quantitative EEG in frontotemporal dementia," Clinical EEG Electroencephalography, vol. 27, no. 2, pp. 61-68, 1996.

[29] J. Dauwels, F. Vialatte, and A. Cichocki, "Diagnosis of Alzheimer's disease from EEG signals: where are we standing?" Current Alzheimer Research, vol. 7, no. 6, pp. 487-505, 2010.

[30] J. Dauwels, F. Vialatte, C. Latchoumane, J. Jeong, and A. Cichocki, "EEG synchrony analysis for early diagnosis of Alzheimer's disease: a study with several synchrony measures and EEG data sets," in Proceedings of the Annual International
Conference of the IEEE Engineering in Medicine and Biology Society, vol. 2009, pp. 2224-2227, 2009.

[31] P. H. Tsai, C. Lin, J. Tsao et al., "Empirical mode decomposition based detrended sample entropy in electroencephalography for Alzheimer's disease," Journal of Neuroscience Methods, vol. 210, no. 2, pp. 230-237, 2012.

[32] J. Jeong, "EEG dynamics in patients with Alzheimer's disease," Clinical Neurophysiology, vol. 115, no. 7, pp. 1490-1505, 2004.

[33] K. J. Stam, D. L. J. Tavy, B. Jelles, H. A. M. Achtereekte, J. P. J. Slaets, and R. W. M. Keunen, "Non-linear dynamical analysis of multichannel EEG: clinical applications in dementia and Parkinson's disease," Brain Topography, vol. 7, no. 2, pp. 141-150, 1994.

[34] C. J. Stam, B. Jelles, H. A. M. Achtereekte, S. A. R. B. Rombouts, J. P. J. Slaets, and R. W. M. Keunen, "Investigation of EEG non-linearity in dementia and Parkinson's disease," Electroencephalography and Clinical Neurophysiology, vol. 95, no. 5, pp. 309-317, 1995.

[35] C. Besthorn, R. Zerfass, C. Geiger-Kabisch et al., "Discrimination of Alzheimer's disease and normal aging by EEG data," Electroencephalography and Clinical Neurophysiology, vol. 103, no. 2, pp. 241-248, 1997.

[36] C.-F. V. Latchoumane, F.-B. Vialatte, J. Solé-Casals et al., "Multiway array decomposition analysis of EEGs in Alzheimer's disease," Journal of Neuroscience Methods, vol. 207, no. 1, pp. 41$50,2012$.

[37] J. Escudero, D. Abasolo, R. Hornero, P. Espino, and M. Lopez, "Analysis of electroencephalograms in Alzheimer's disease patients with multiscale entropy," Physiological Measurement, vol. 27, no. 11, pp. 1091-1106, 2006.

[38] T. Mizuno, T. Takahashi, R. Y. Cho et al., "Assessment of EEG dynamical complexity in Alzheimer's disease using multiscale entropy," Clinical Neurophysiology, vol. 121, no. 9, pp. 1438-1446, 2010.

[39] C.-K. Peng, S. Havlin, H. E. Stanley, and A. L. Goldberger, "Quantification of scaling exponents and crossover phenomena in nonstationary heartbeat time series," Chaos, vol. 5, no. 1, pp. 82-87, 1995.

[40] Y.-L. Ho, C. Lin, Y.-H. Lin, and M.-T. Lo, “The prognostic value of non-linear analysis of heart rate variability in patients with congestive heart failure-a pilot study of multiscale entropy," PLoS ONE, vol. 6, no. 4, Article ID e18699, 2011.

[41] P. C. Ivanov, K. Hu, M. F. Hilton, S. A. Shea, and H. E. Stanley, "Endogenous circadian rhythm in human motor activity uncoupled from circadian influences on cardiac dynamics," Proceedings of the National Academy of Sciences of the United States of America, vol. 104, no. 52, pp. 20702-20707, 2007.

[42] K. Hu, E. J. W. van Someren, S. A. Shea, and F. A. J. L. Scheer, "Reduction of scale invariance of activity fluctuations with aging and Alzheimer's disease: involvement of the circadian pacemaker," Proceedings of the National Academy of Sciences of the United States of America, vol. 106, no. 8, pp. 2490-2494, 2009.

[43] K. Hu, F. A. J. L. Scheer, P. C. Ivanov, R. M. Buijs, and S. A. Shea, "The suprachiasmatic nucleus functions beyond circadian rhythm generation," Neuroscience, vol. 149, no. 3, pp. 508-517, 2007.

[44] M. Costa, A. L. Goldberger, and C.-K. Peng, "Multiscale entropy analysis of complex physiologic time series," Physical Review Letters, vol. 89, no. 6, Article ID 068102, 2002.

[45] M. Costa, A. L. Goldberger, and C.-K. Peng, "Multiscale entropy analysis of biological signals," Physical Review E. Statistical, 
Nonlinear, and Soft Matter Physics, vol. 71, no. 2, Article ID 021906, 2005.

[46] H.-K. Yuan, C. Lin, P.-H. Tsai et al., "Acute increase of complexity in the neurocardiovascular dynamics following carotid stenting," Acta Neurologica Scandinavica, vol. 123, no. 3, pp. 187192, 2011.

[47] P. J. Pan, P. H. Tsai, C. C. Tsai, C. L. Chou, M. T. Lo, and J. H. Chiu, "Clinical response and autonomic modulation as seen in heart rate variability in mechanical intermittent cervical traction: a pilot study," Journal of Rehabilitation Medicine, vol. 44, no. 3, pp. 229-234, 2012.

[48] L. Descarries, V. Gisiger, and M. Steriade, "Diffuse transmission by acetylcholine in the CNS," Progress in Neurobiology, vol. 53, no. 5, pp. 603-625, 1997.

[49] J. Matthews-Bellinger and M. M. Salpeter, "Distribution of acetylcholine receptors at frog neuromuscular junctions with a discussion of some physiological implications," The Journal of Physiology, vol. 279, pp. 197-213, 1978.

[50] A. Scimemi and M. Beato, "Determining the neurotransmitter concentration profile at active synapses," Molecular Neurobiology, vol. 40, no. 3, pp. 289-306, 2009.

[51] M. F. Folstein, S. E. Folstein, and P. R. McHugh, "'Mini-mental state. A practical method for grading the cognitive state of patients for the clinician," Journal of Psychiatric Research, vol. 12, no. 3, pp. 189-198, 1975.

[52] C. P. Hughes, L. Berg, and W. L. Danziger, "A new clinical scale for the staging of dementia," British Journal of Psychiatry, vol. 140, no. 6, pp. 566-572, 1982.

[53] J. E. Cooper, "On the publication of the diagnostic and statistical manual of mental disorders: fourth edition (DSM-IV)," The British Journal of Psychiatry, vol. 166, no. 1, pp. 4-8, 1995.

[54] M. R. Nuwer, "Assessing digital and quantitative EEG in clinical settings," Journal of Clinical Neurophysiology, vol. 15, no. 6, pp. 458-463, 1998.

[55] N. E. Huang, Z. Shen, S. R. Long et al., "The empirical mode decomposition and the Hilbert spectrum for nonlinear and non-stationary time series analysis," The Royal Society of London. Proceedings, Series A: Mathematical, Physical and Engineering Sciences, vol. 454, no. 1971, pp. 903-995, 1998.

[56] S. Pincus, "Approximate entropy (ApEn) as a complexity measure," Chaos, vol. 5, no. 1, pp. 110-117, 1995.

[57] S. M. Pincus, "Approximate entropy as a measure of system complexity," Proceedings of the National Academy of Sciences of the United States of America, vol. 88, no. 6, pp. 2297-2301, 1991.

[58] J. S. Richman and J. R. Moorman, "Physiological time-series analysis using approximate entropy and sample entropy," American Journal of Physiology-Heart and Circulatory Physiology, vol. 278, no. 6, pp. H2039-H2049, 2000.

[59] B. Katz and R. Miledi, “The measurement of synaptic delay, and the time course of acetylcholine release at the neuromuscular junction," Proceedings of the Royal Society of London Series B, vol. 161, pp. 483-495, 1965. 


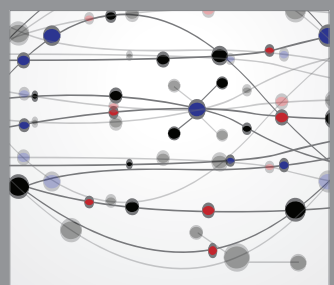

The Scientific World Journal
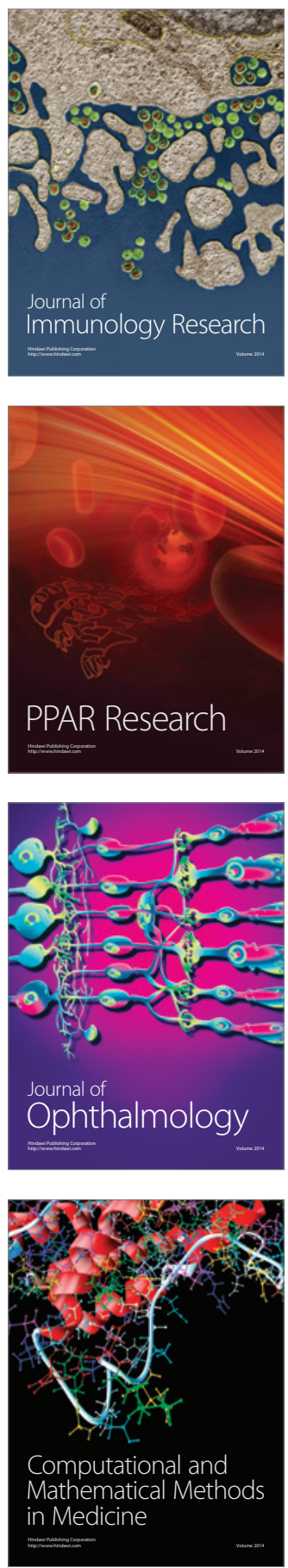

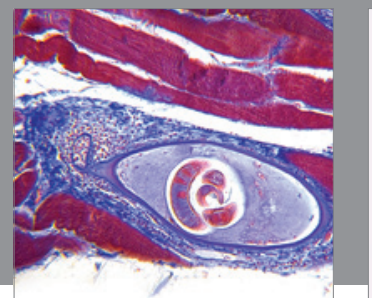

Gastroenterology

Research and Practice
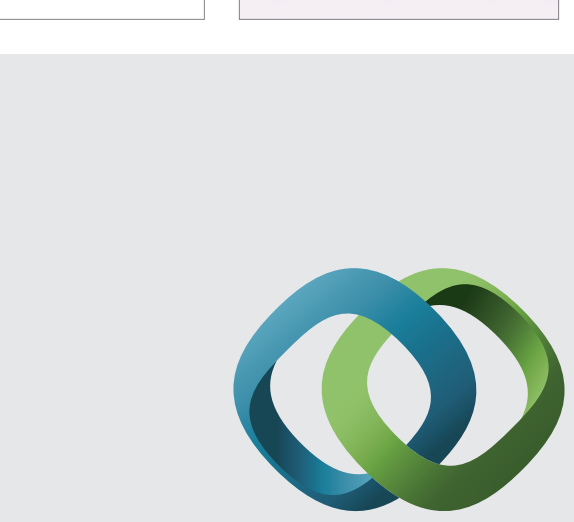

\section{Hindawi}

Submit your manuscripts at

http://www.hindawi.com
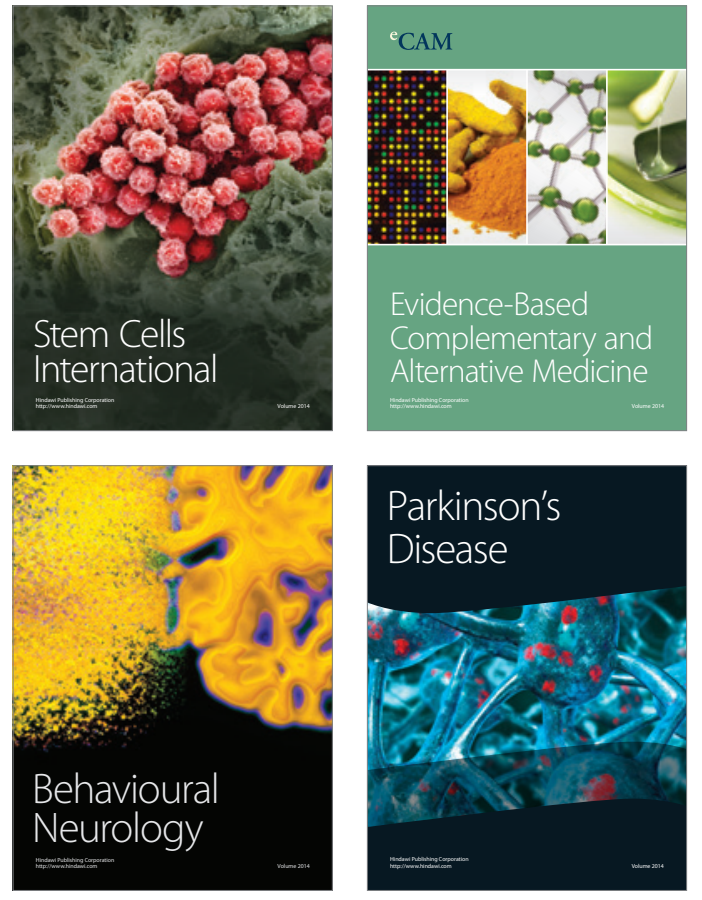
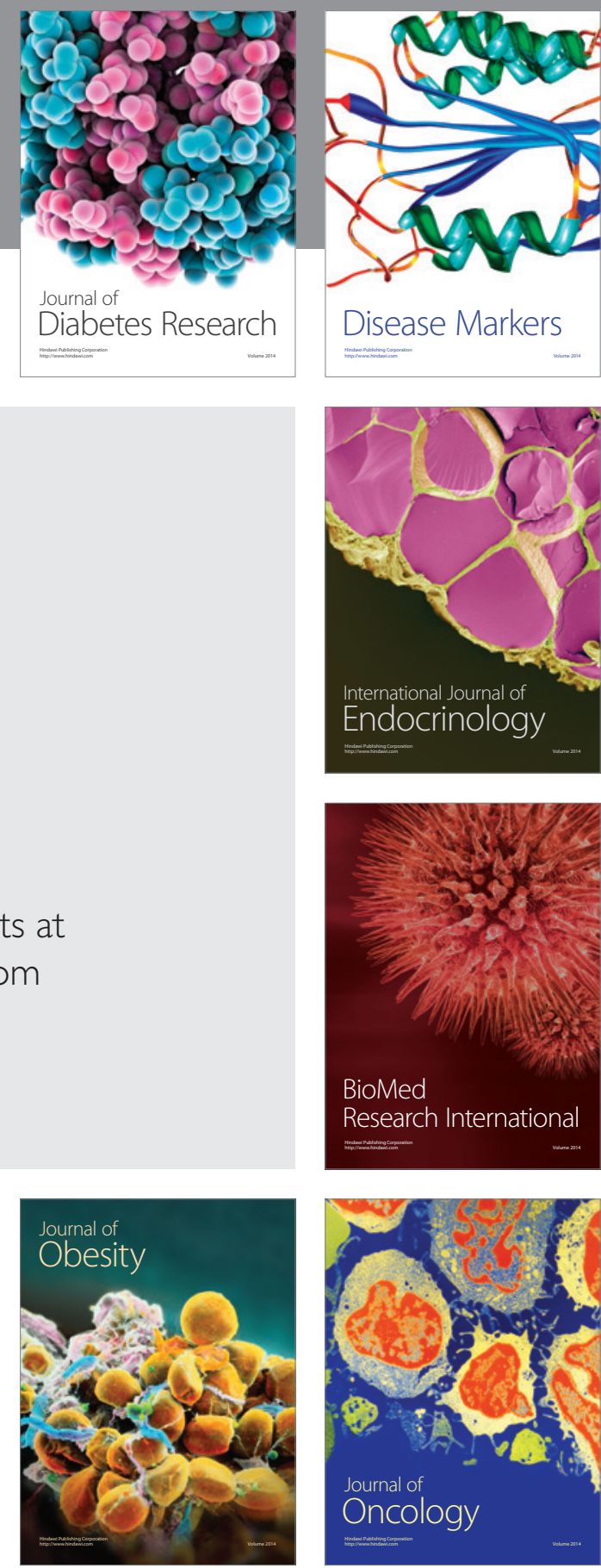

Disease Markers
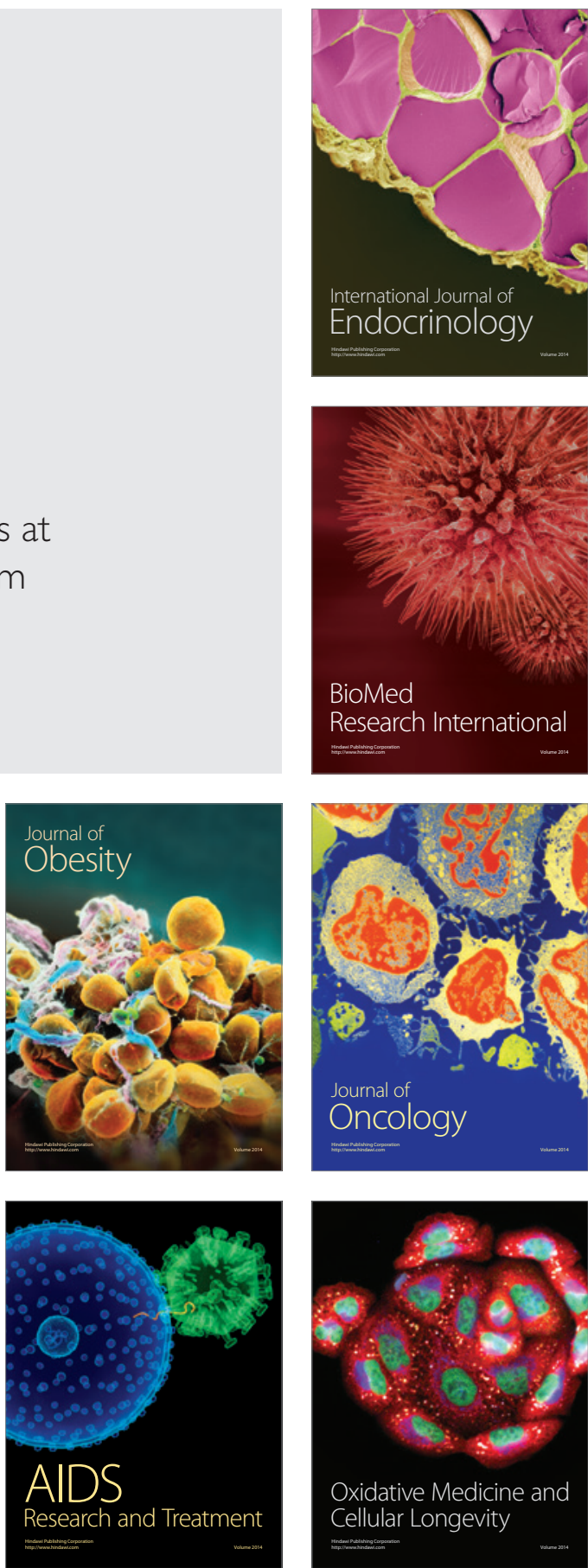